\title{
A pneumatically driven inkjet printing system for highly viscous microdroplet formation
}

\author{
In Ho Choi and Joonwon Kim ${ }^{*}$
}

\begin{abstract}
This paper introduces a pneumatically driven inkjet printing system that forms highly viscous microdroplets in the nanoliter volume range. The printing system has a unique printing mechanism that uses a flexible membrane and an effective backflow stopper. While typical inkjet systems can handle liquids with a limited range of viscosity due to energy loss by viscous dissipation at the nozzle and ineffective backflow management within their systems, our printing system can print liquids with viscosity as high as $384.5 \mathrm{CP}$. In the viscosity range 1-384.5 CP, we investigated printing characteristics such as printed droplet volume, standoff distance, and maximum possible frequency. The droplet formation showed outstanding reliability, with the droplet volume exhibiting a coefficient of variation less than $1.07 \%$. Our printing system can be directly used in inkjet applications with functional liquids over a broad viscosity range.
\end{abstract}

Keywords: Drop-on-demand inkjet, Droplet formation, High viscosity, Pneumatic actuation, Printing mechanism

\section{Background}

Inkjet technology, which delivers a minute droplet onto a desired position, offers several advantages for fabricating micropatterns, including reduced material usage, minimized use of hazardous etching chemicals, compatibility with various substrates, and reduced number of process steps [1]. Thus, inkjet technology has been regarded as an effective fabrication tool for the use of liquid functional materials and has been implemented into research areas such as fabrication of organic thin-film transistors [2], organic solar cells [3], 3D structures [4], and cellular structures [5]. The properties (i.e., viscosity, surface tension, and density) of the printing liquids govern the operation of an inkjet printing system. Among these properties, viscosity is often a limiting factor because the ejection of the droplets depends on the amount of energy loss caused by viscous dissipation at the nozzle $[6,7]$. In general, the viscosity must be less than $30 \mathrm{cP}$

\footnotetext{
*Correspondence: joonwon@postech.ac.kr

Department of Mechanical Engineering, Pohang University of Science and Technology (POSTECH), 77 Cheongam-Ro, Nam-Gu, Pohang, Gyeongbuk 37673, Republic of Korea
}

[8]. Because of the viscosity limitation, many researchers have attempted to develop appropriate functional liquids for use in inkjet systems (e.g., lowering viscosity via dilution). The limited viscosity of the printing liquids restricts the content of functional pigments, leading to limited functionality (i.e., the printing liquids in an inkjet system can carry a lower amount of functional pigments than those in a contact printing system) [9]. Thus, contact printing methods such as screen and offset printings are preferred for handling highly viscous functional liquids. If the allowable viscosity range for inkjet systems can be increased, the printed results will exhibit greater performance and better throughput. Therefore, to extend the range of applications of inkjet printing, it is essential to develop a printing mechanism that can eject viscous liquids as well as appropriate printing liquids.

In this study, we present an inkjet printing system that forms highly viscous microdroplets in the nanoliter volume range. To print highly viscous liquids, a unique pneumatically driven printing mechanism with a flexible membrane and an effective backflow stopper was used in the printing head. Since the effective backflow stopper can provide complete confinement of the liquid within 
the chamber along with the flexible membrane, energy loss during the ejection of the droplets can be dramatically reduced. Therefore, effective energy transfer can be realized which enables highly viscous microdroplet formation.

The performance of the printing system was assessed by application of liquids with a broad range of viscosity $(1-384.5 \mathrm{cP})$. The system showed reliable performance, with the droplet volume exhibiting a coefficient of variation $(\mathrm{CV})$ less than $1.07 \%$. For analyzing printing characteristics, the dependence of the printing volume, minimum standoff distance, and maximum printing frequency on the liquid viscosity was examined. While typical inkjet systems can use liquids with a limited range of viscosity, our printing system can print liquids of viscosity as high as $384.5 \mathrm{cP}$ without additional energy (e.g., heating of printing liquids to reduce viscosity). Therefore, our printing system can be directly used in inkjet applications with functional liquids over a broad viscosity range, without a need for controlling viscosity of printing liquids.

\section{Printing mechanism}

The printing module of our pneumatic printing system includes a printing head to generate droplets, a liquid reservoir to supply and store liquid into the printing head, and an operating part with a solenoid valve to switch pressures on the printing head (Fig. 1a). Each part of the printing head was fabricated using the microelectromechanical systems (MEMS) fabrication processes. In the printing head, a flexible membrane is located between rigid substrates comprising a silicon layer and a glass layer. Using deep reactive-ion etching (DRIE) process, the silicon layer was designed to have a nozzle (length $100 \mu \mathrm{m}$ length, diameter $100 \mu \mathrm{m}$ ), a chamber (diameter $1.5 \mathrm{~mm}$, height $100 \mu \mathrm{m}$ ), and a donut-shaped bump structure (inner diameter $1.5 \mathrm{~mm}$, outer diameter $2.5 \mathrm{~mm}$ ) to prevent backflow. The through-hole patterns in the glass layer were formed by a wet etching process using hydrogen fluoride. The diameters of the inlet hole and the actuating holes are 2 and $3 \mathrm{~mm}$, respectively. The flexible membrane (thickness $70 \mu \mathrm{m}$ ) was obtained by spin coating with a 10:1 (weight ratio) mixture of a polydimethylsiloxane (PDMS) prepolymer and a curing agent. For assembly, all parts were bonded using an $\mathrm{O}_{2}$ plasma treatment. For stable droplet formation, a hydrophobic coating of $1 \%$ Teflon solution was applied on the outside of the nozzle after assembly.

To eject microdroplets, the printing head is actuated by positive and negative pressures transferred during the on and off times of the solenoid valve, which is triggered by electric signals tailored from a LabVIEW program (Fig. 2). When negative pressure is applied onto the flexible membrane in the head, the closed chamber is opened by the membrane deflected upward and is filled with liquid from the reservoir (Fig. 2a). Then, positive pressure is applied onto the membrane and the chamber is instantly closed to prevent backflow, which is an unwanted flow toward the inlet of the printing head (Fig. 2b). The membrane deflected downward by further positive pressure squeezes the chamber to generate a droplet (Fig. 2c). A microdroplet is formed per electric signal pulse transferred to the solenoid valve in a dropon-demand (DOD) mode. The droplet formation process is repeated for several microdroplets.

\section{Experiment setup}

Our experiment setup consists of a printing system, an in situ monitoring system, and a measuring system for analyzing the droplet formation and printing volume (Fig. 3). The printing system consists of the printing module, a laboratory-built pneumatic controller (which consistently provides positive and negative pressures at set values ranging from -100 to $200 \mathrm{kPa}$ ), and a computer for running the LabVIEW program. The in situ monitoring system consists of a high-speed camera (Phantom MIRO M320S, Vision Research) and an LED light source. The droplet formation process was captured at $15,000 \mathrm{fps}$. The droplets were collected in a silicone oil bath to prevent evaporation and weighed using a precision scale with a resolution of $0.0001 \mathrm{~g}$ (MS-70, A\&D Ltd.); the mass thus measured was converted into the droplet volume [10].

To confirm the printable viscosity range of our system, printing liquids with different viscosity values were prepared using glycerol and deionized (DI) water. Figure 4 shows the variation in the viscosity of the glycerol-water mixture with glycerol concentration in terms of weight ratio. The viscosity exponentially increases with glycerol concentration over a small surface tension variation. Glycerol-water mixture concentrations of 0, 40, 60, 80, 84,88 , and $92 \%$ glycerol-water mixtures were used for the experiment; these values correspond to 1.0, 3.7, 10.7, $59.9,84.3,147.5$, and $384.5 \mathrm{cP}$, respectively [11].

\section{Results and discussion \\ Operating conditions}

The conditions for each printing liquid, such as the magnitudes of positive and negative pressures, the push time for deflecting the membrane downward by positive pressure, and the pull time for deflecting the membrane upward by negative pressure, were optimized to operate the printing head. To generate a droplet, an appropriate kinetic energy must be transferred to the liquid. The kinetic energy is adjusted by positive pressure and push time. The higher the viscosity of the printing liquid, the 


\section{a}

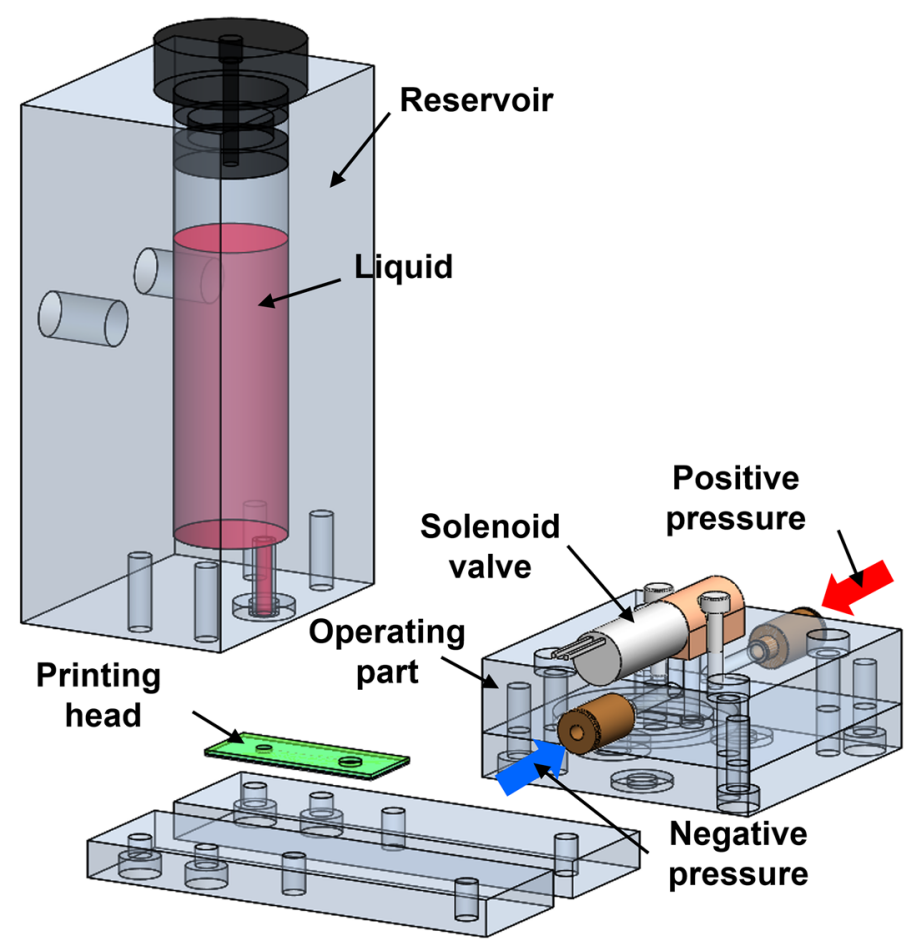

b

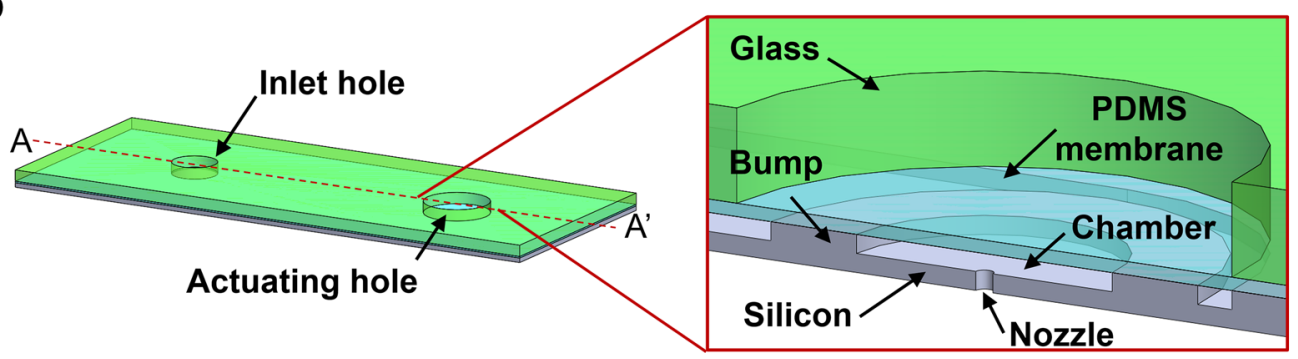

Fig. 1 Overview of printing module: a disassembly of printing module and $\mathbf{b}$ printing head

higher the energy required to generate a droplet. For glycerol-water mixture concentrations of $0,40,60,80,84,88$, and $92 \%$, the required minimum positive pressures to eject a minimum volume of each viscous liquid were 2,3 , $10,65,150,200$, and $200 \mathrm{kPa}$, respectively, at a fixed push time of $3 \mathrm{~ms}$. In the case of the $92 \%$ glycerol-water mixture, a higher push time of $5 \mathrm{~ms}$ was set to transfer more energy because $200 \mathrm{kPa}$ is the maximum pressure in our current configuration. Negative pressure is optimized to prevent air bubble entrapment in the chamber of the printing head and was fixed at $-5 \mathrm{kPa}$ for all printing liquids. For each printing liquid, sufficient pull times were provided within $600 \mathrm{~ms}$ to return the liquid meniscus at the end of the nozzle to its initial state. The height of the liquid column in the reservoir was maintained at $40 \mathrm{~mm}$ for each test.

\section{Droplet formation}

Successful droplet formation occurred for all printing liquids in the viscosity range $1-384.5 \mathrm{cP}$, as observed by the in situ monitoring system (Fig. 5). Adequate kinetic energy should be transferred to the liquid to overcome the energy loss at the end of the nozzle for viscous droplet formation. To minimize the energy loss, the actuating area and the $100 \mu \mathrm{m}$ diameter nozzle are concentrically aligned to minimize flow resistance. In the printing mechanism, high positive pressure up to $200 \mathrm{kPa}$ can be directly applied onto the thin flexible membrane. The bump structure, which acts as a backflow stopper by closing the chamber with the deflected membrane by positive pressure, inhibits an undesirable backflow toward the inlet and effectively transfers kinetic energy only toward the nozzle (Fig. 2b). In addition to the efficient 

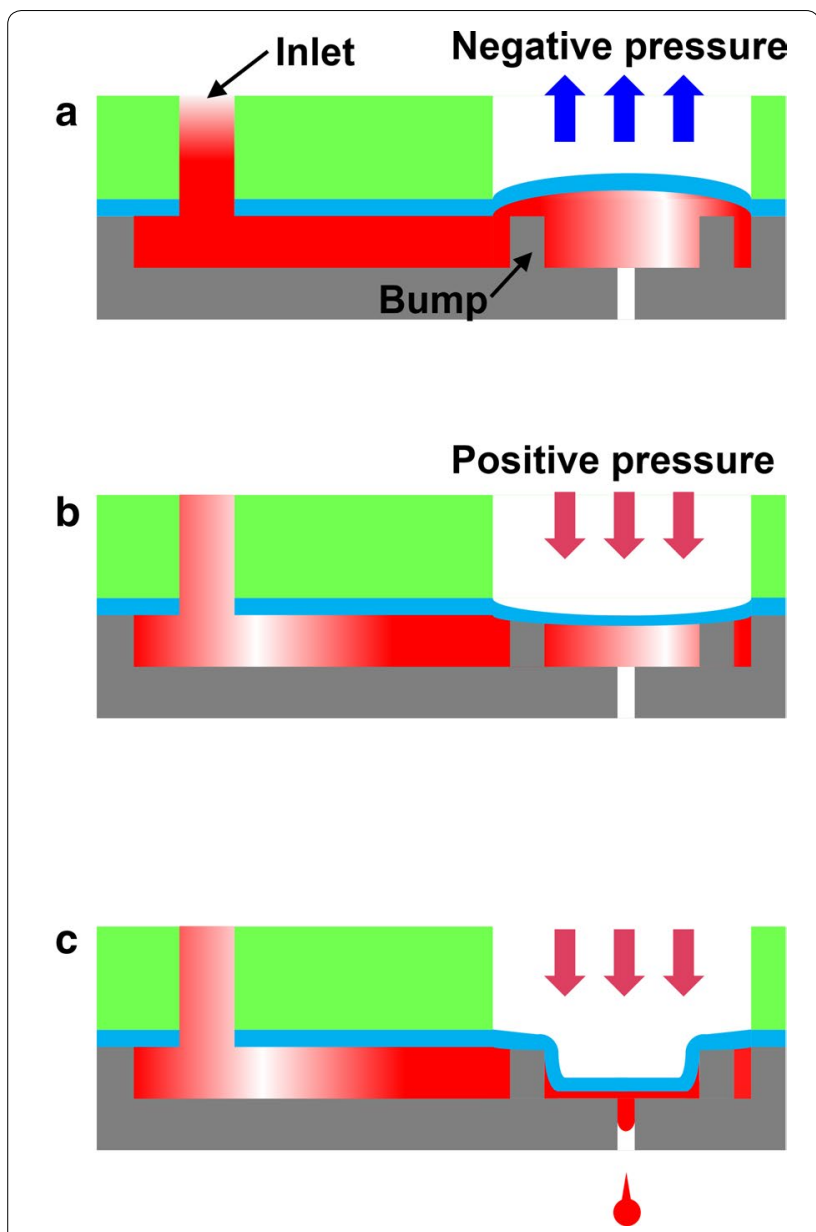

Fig. 2 Printing mechanism for droplet formation in the view A-A' in Fig. 1 b: a liquid filling, b prevention of backflow by bump structure, and $\mathbf{c}$ liquid ejection

energy transfer, when the printing liquid has high viscosity, the liquid is continually stretched by inertial motion of the liquid during the pull time to apply negative pressure onto the membrane after push time. The negative pressure promotes liquid necking during the stretching stage (see the droplet formation images after 3 and $5 \mathrm{~ms}$ in Fig. 5b, c respectively). This necking process can be observed for glycerol-water mixtures with the glycerol concentration higher than $80 \%$.

Numerous droplets (over 10,000) were weighed to measure the printed droplet volume. The printed volume at the operating condition gradually increased with glycerol concentration because a high-viscosity liquid requires a high positive pressure due to the increased energy loss caused by viscous dissipation at the nozzle (Fig. 6). In the printing mechanism, the amount of the energy transferred to the liquid in the chamber of the head is determined by the magnitude of the positive pressure applied to the membrane during the push time. A higher positive pressure causes more volume in the chamber to be squeezed by the deflected membrane. The squeezed liquid in the chamber stretches through the nozzle, forming a droplet. The printing system generated droplets at a precise volume. The measured droplet volumes were $12.2(\mathrm{CV}=1.02 \%), 13.0(\mathrm{CV}=0.79 \%), 14.9$ $(\mathrm{CV}=1.04 \%), 34.1(\mathrm{CV}=0.83 \%), 41.6(\mathrm{CV}=0.97 \%)$, $45.8(\mathrm{CV}=1.07 \%)$, and $63.5(\mathrm{CV}=0.79 \%)$ for 20,40 , $60,80,84,88$, and $92 \%$ glycerol-water mixtures, respectively. The performance of our system is reliable because the mean values of the measured droplet volume exhibited a CV less than $1.07 \%$. In the current printing setup, our system prints a droplet of $63.5 \mathrm{~nL}$ at $384 \mathrm{cP}$. To achieve a smaller droplet volume range, the nozzle size can be reduced; however, narrower nozzles induce more energy loss. Therefore, a higher performance pump that can apply a higher pressure is essential to transfer higher energy as the nozzle diameter decreases.

\section{Minimum standoff distance}

Inkjet printing is categorized as a noncontact fabrication method. The space between the nozzle and the substrate must be secured and is called the "standoff distance." The minimum standoff distance is defined as the distance at which a stretched liquid from the nozzle merges into a single droplet with satellite droplets. Printing at a shorter standoff distance than the minimum standoff distance degrades the quality of the printing result. A longer standoff distance produces an inaccurately printed pattern due to the undesired airflow in a printing setting. Thus, we analyzed the minimum standoff distance of our system for the various liquids examined (Fig. 7). The minimum standoff distance increases on increasing the glycerol concentration from 40 to $92 \%$ because high viscosity requires high kinetic energy, causing a long tail behind the main head of the liquid filament. Distances of $0.62 \pm 0.02,0.67 \pm 0.03,2.08 \pm 0.15,2.85 \pm 0.11$, and $3.51 \pm 0.06 \mathrm{~mm}$ were required for $40,60,80,84,88$, and $92 \%$ glycerol-water mixtures, respectively. From this trend, a distance lower than $0.62 \mathrm{~mm}$ would be expected for DI water (0 \% glycerol concentration). However, a longer than expected standoff distance of $1.09 \pm 0.03 \mathrm{~mm}$ was required. For the $0 \%$ glycerol concentration liquid, the liquid droplet is formed at the positive pressure of $2 \mathrm{kPa}$, the minimum pressure required to form a droplet. However, a greater amount of energy than that required to form a single droplet was transferred although a relatively lower positive pressure was applied. Thus, a long filament with a high aspect ratio was formed, causing the formation of satellites. The satellite droplet formation during the single droplet formation was observed via in situ monitoring (Fig. 5a). Although the minimum standoff distance to preserve an inaccurately printed 


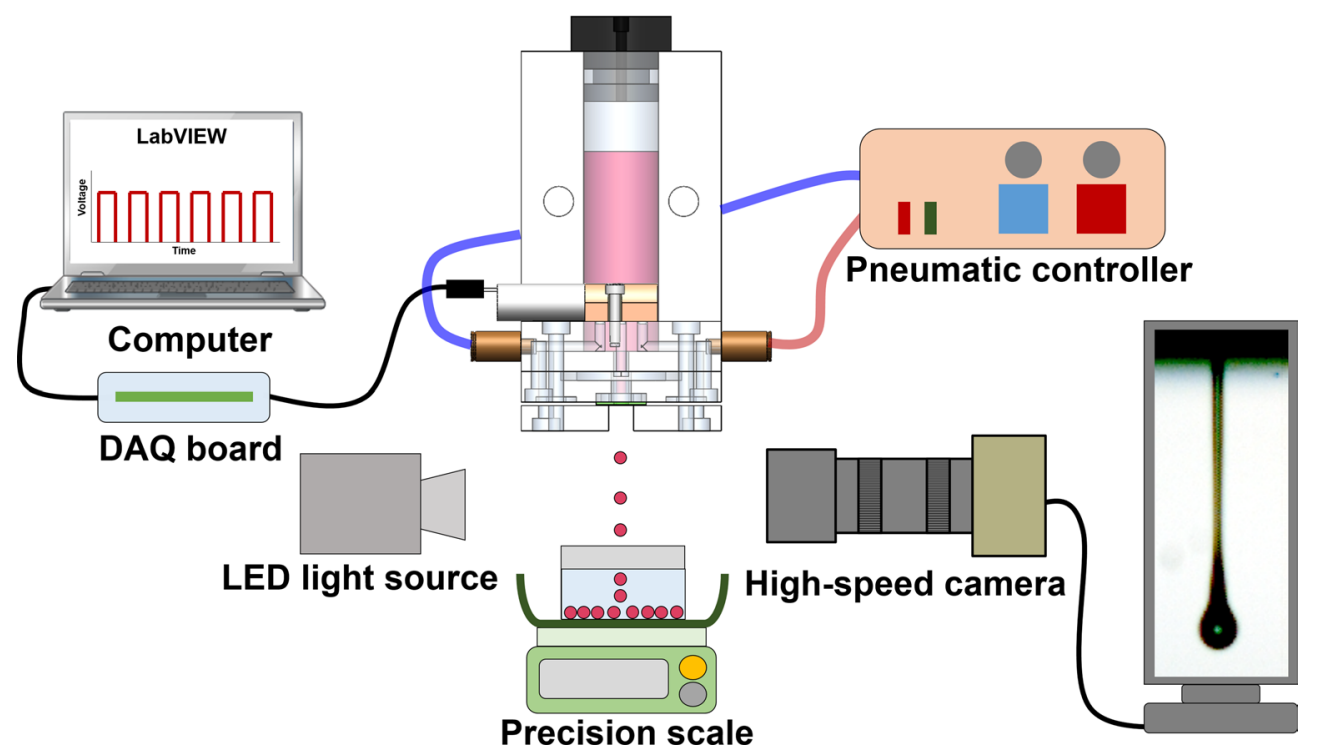

Fig. 3 Experimental setup for monitoring droplet formation and measuring printed droplet volume

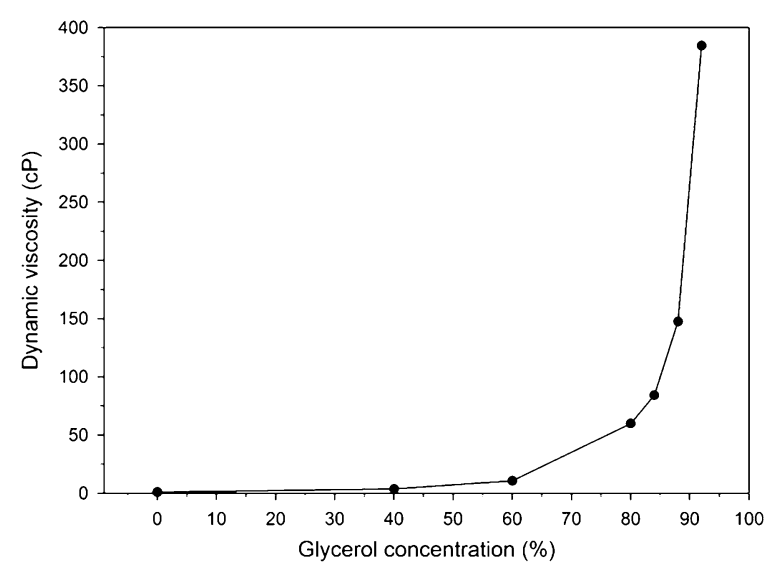

Fig. 4 Viscosity variation with glycerol concentration (wt.\%) for glycerol-water mixtures

pattern caused by undesired airflow in a printing setting is within $1 \mathrm{~mm}$ of the value for typical inkjet systems [12], our system has longer standoff distances at different viscosities, which indicates that our system is compatible with various structured substrates.

\section{Droplet formation frequency}

The printing frequency is a parameter that is correlated with fabrication throughput. The liquid properties of printing liquids and printing actuating types restrict the maximum printing frequency. For a given printing head design, the maximum potential frequency is determined by the inverse of the time taken for formation of a droplet [7]. The ideal time to form a droplet is estimated via repeated in situ monitoring (Fig. 8). With increasing glycerol concentration, the time to eject a droplet and prepare for the next droplet formation increases: The $0,40,60,80,84,88$, and $92 \%$ glycerol-water mixtures required a time of $2.84 \pm 0.08,5.21 \pm 0.20$, $10.67 \pm 0.38,39.24 \pm 0.9185 .95 \pm 0.59,145.81 \pm 1.21$, and $225.55 \pm 2.65 \mathrm{~ms}$, respectively. The averaged time for each liquid can be converted to the maximum possible frequency (Fig. 8). Because a longer time is required to form a droplet for a more viscous liquid, the maximum frequency is low at high viscosity: The maximum frequency for the $0,40,60,80,84,88$, and $92 \%$ glycerolwater mixtures was $352.11,191.82$, 93.75, 25.48, 11.64, 6.86 , and $4.43 \mathrm{~Hz}$, respectively. During the droplet formation process for high-viscosity liquids, the residual liquid after pinch-off of the main droplet in a filament required a long time to return to its initial state to prepare for the next droplet formation (see the formation process after $4.5 \mathrm{~ms}$ in Fig. $5 \mathrm{~b}$ and $12.7 \mathrm{~ms}$ in Fig. $5 \mathrm{c}$ ). In the whole process time, most of the time is spent on retraction of the residual liquid. To reduce the retraction time, the friction between the hanging liquid and the outside surface of the nozzle should be minimized. The maximum frequency can be improved by the application of a superhydrophobic surface on the outside of the nozzle. 

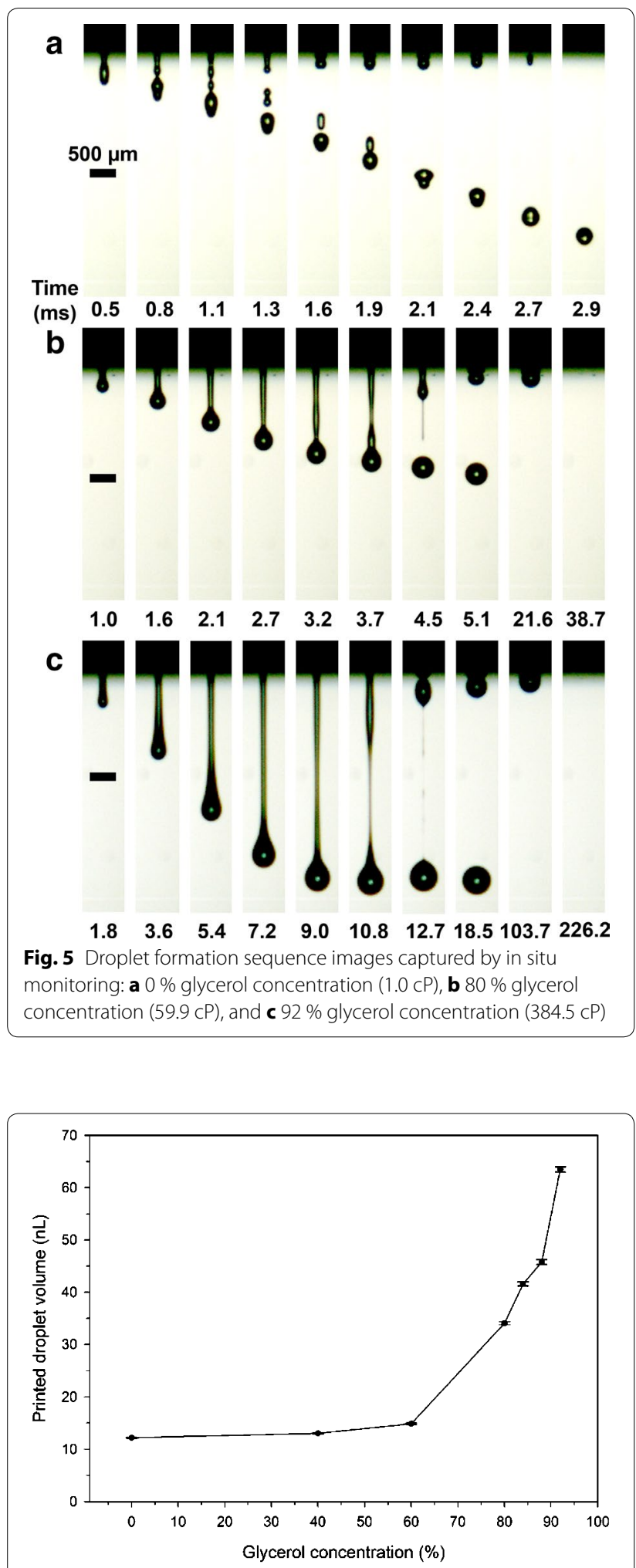

Fig. 6 Variation of the minimum printed droplet volumes with glycerol concentration



Fig. 7 Variation of the measured minimum standoff distances to form a droplet with glycerol concentration

\section{Conclusions}

We developed a pneumatically driven inkjet printing system that forms highly viscous microdroplets in the nanoliter volume range. The performance of the printing system at high viscosity was assessed via the application of liquids with a broad range of viscosity $(1-384.5 \mathrm{cP})$. The operating conditions were optimized to determine the minimum droplet volumes for each liquid. The printed droplet volumes were in the range $12.2-63.5 \mathrm{~nL}$ and increased with the viscosity of the printing liquids. The printing system showed outstanding reliability in droplet formation, with a $\mathrm{CV} \leq 1.07 \%$ for the droplet volume. The minimum standoff distance was in the range $1.09-3.51 \mathrm{~mm}$ and depended on the viscosity of the printing liquids. The standoff distance was increased by high viscosity and excessive energy, leading to long tails during droplet formation. The printing frequency was estimated as the time to form a droplet and was found to be strongly affected by the high viscosity of the printing liquids. Liquids with high viscosity required a long retraction time (i.e., the time taken by the liquid to return to its initial state) for the residual liquid at the end of the nozzle after pinch-off. While typical inkjet systems can print liquids with a limited viscosity range, our printing system can print liquids of viscosity ranging from 1 to $384.5 \mathrm{cP}$ without the need for additional energy (e.g., heating of printing liquids to reduce the viscosity). Therefore, without requiring viscosity control of the liquids, our printing system can be directly used in inkjet applications with functional liquids over a broad viscosity range. 


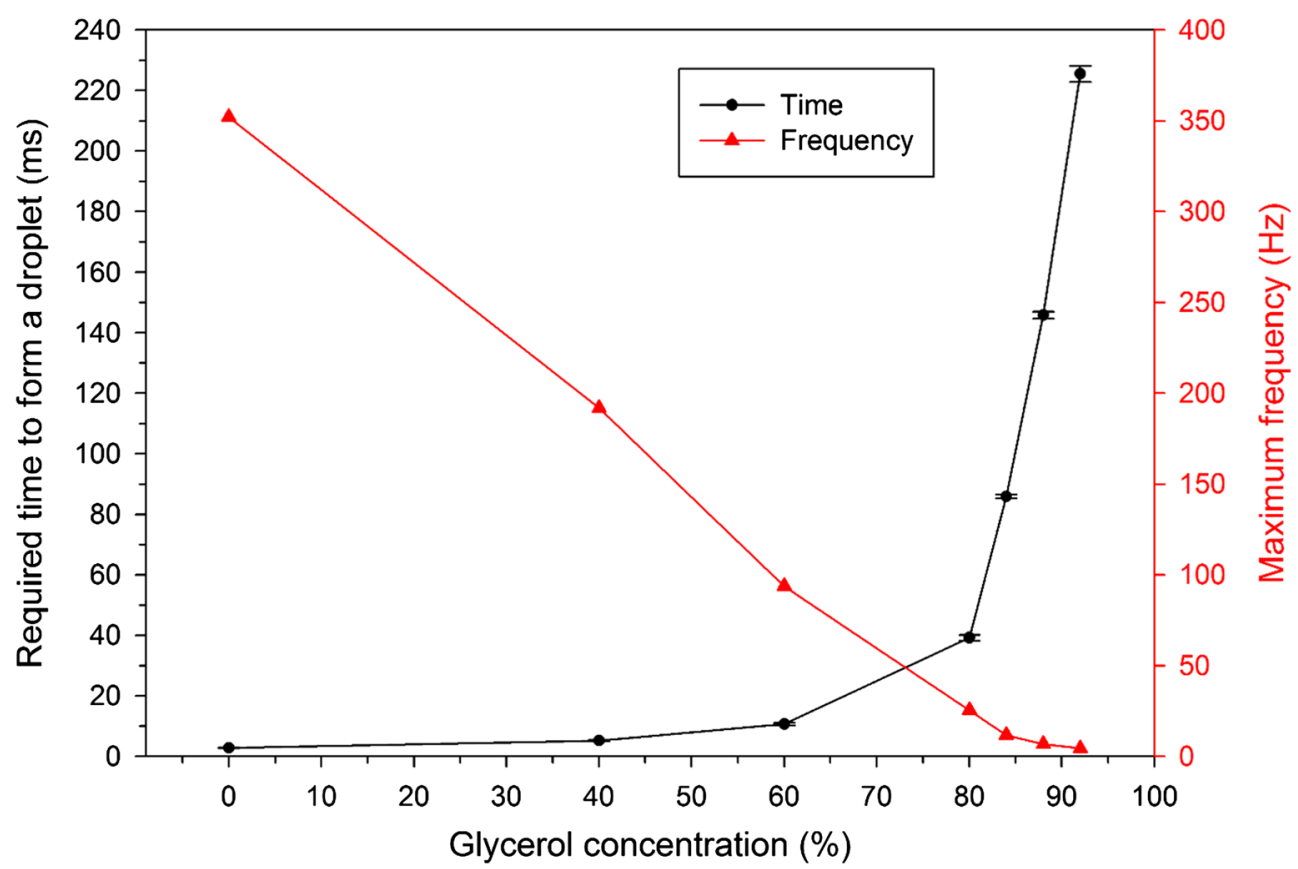

Fig. 8 Variation of required times to form a droplet and estimated maximum frequencies with glycerol concentration

\section{Authors' contributions}

IHC participated in design and fabrication, carried the experiments, contributed to the data analysis and drafted the manuscript. JK conceived of the study, participated in its design and coordination, reviewed all test results, and finalized the drafted manuscript. Both authors read and approved the final manuscript.

\section{Acknowledgements}

This research was supported by a Grant of the Korea Health Technology R\&D Project through the Korea Health Industry Development Institute (KHIDI), funded by the Ministry of Health \& Welfare, Republic of Korea (Grant number: HI15C0001).

\section{Competing interests}

The authors declare that they have no competing interests.

Received: 7 April 2016 Accepted: 1 June 2016

Published online: 09 June 2016

\section{References}

1. Tekin E, Smith PJ, Schubert US (2008) Inkjet printing as a deposition and patterning tool for polymers and inorganic particles. Soft Matter 4(4):703-713. doi:10.1039/B711984D

2. Kwak D, Lim JA, Kang B, Lee WH, Cho K (2013) Self-organization of inkjetprinted organic semiconductor films prepared in inkjet-etched microwells. Adv Funct Mater 23(42):5224-5231. doi:10.1002/adfm.201300936
3. Kim YH, Sachse C, Machala ML, May C, Müller-Meskamp L, Leo K (2011) Highly conductive PEDOT:PSS electrode with optimized solvent and thermal post-treatment for ITO-free organic solar cells. Adv Funct Mater 21(6):1076-1081. doi:10.1002/adfm.201002290

4. Kröber P, Delaney JT, Perelaer J, Schubert US (2009) Reactive inkjet printing of polyurethanes. J Mater Chem 19(29):5234. doi:10.1039/b823135d

5. Di Biase M, Saunders RE, Tirelli N, Derby B (2011) Inkjet printing and cell seeding thermoreversible photocurable gel structures. Soft Matter 7(6):2639-2646. doi:10.1039/COSM00996B

6. Derby B (2010) Inkjet printing of functional and structural materials: fluid property requirements, feature stability, and resolution. Annu Rev Mater Res 40(1):395-414. doi:10.1146/annurev-matsci-070909-104502

7. Jang D, Kim D, Moon J (2009) Influence of fluid physical properties on inkjet printability. Langmuir 25(5):2629-2635. doi:10.1021/la900059m

8. Krebs FC (2009) Fabrication and processing of polymer solar cells: a review of printing and coating techniques. Sol Energy Mater Sol Cells 93(4):394-412. doi:10.1016/j.solmat.2008.10.004

9. Castrejon-Pita JR, Baxter WRS, Morgan J, Temple S, Martin GD, Hutchings IM (2013) Future, opportunities and challenges of inkjet technologies. Atomization Sprays 23(6):541-565. doi:10.1615/AtomizSpr.007653

10. Verkouteren RM, Verkouteren JR (2009) Inkjet metrology: high-accuracy mass measurements of microdroplets produced by a drop-on-demand dispenser. Anal Chem 81(20):8577-8584. doi:10.1021/ac901563j

11. Lide DR (2005) CRC handbook of chemistry and physics (86th edn). Taylor \& Francis Group, Boca Raton

12. Hoath SD, Harlen OG, Hutchings IM (2012) Jetting behavior of polymer solutions in drop-on-demand inkjet printing. J Rheol 56(5):1109. doi:10.1122/1.4724331 